\title{
Prognostic Factor of Ureum and Creatinine Serum of Acute Post Streptococcal Glomerulonephritis in Children
}

\author{
Ahmad Hadiwijaya*, Husein Albar, Syarifuddin Rauf, Dasril Daud \\ Department of Pediatrics, Medical Faculty, Hasanuddin University, Makassar, Indonesia \\ Email address: \\ ahmadhadiwijaya0@gmail.com (A. Hadiwijaya), huseinalbar@yahoo.com (H. Albar), hi_unhashospitalpediatrics@yahoo.com (S. Rauf), \\ drdasri@gmail.com (D. Daud)
}

\section{To cite this article:}

Ahmad Hadiwijaya, Husein Albar, Syarifuddin Rauf, Dasril Daud. Prognostic Factor of Ureum and Creatinine Serum of Acute Post Streptococcal Glomerulonephritis in Children. American Journal of Health Research. Vol. 3, No. 3, 2015, pp. 151-155.

doi: 10.11648/j.ajhr.20150303.17

\begin{abstract}
Introduction: Acute post streptococcal glomerulonephritis is one of the commonest causes of glomerular disease in developing countries. Ureum and creatinine examination not only can be used to support the diagnosis but also to identify and detect early organs disturbances and predict the patient prognosis. Objective: This study aims to observe the ureum and creatinine serum level in APSGN children and its relationship with the outcomes. Methods: A retrospective cohort design regarding the prognostic value of serum ureum and creatinine levels for APSGN children outcomes. The data was collected from medical records of APSGN children treated at Dr. Wahidin Sudirohusodo Hospital Makassar from 2009-2013. Results: There is no significant correlation between the outcome and sex $(p=0.961)$, nutritional status $(p=1.000)$, and age $(p=0.108)$. There is a significant difference both in early ureum levels to outcome with $\mathrm{p}=0.003$ and in early creatinine levels to outcome with $p=0.020$. There is tendency to decreased levels of ureum and creatinine serum of survival group ( $p=0.000$ ) while hospitalization and trend of increased or stagnan levels of ureum and creatinine serum of non survival group while hospitalization. Multivariate analyses indicated that serum ureum levels $<119 \mathrm{mg} / \mathrm{dl}$ is an independent prognostic factor for APSGN outcomes in children with $\mathrm{p}=0.032$, OR 1.021, and CI 95\% 1.002-1.041. Conclusion: It seems that serum ureum is an independent prognostic factor for APSGN, where serum ureum levels $<119 \mathrm{mg} / \mathrm{dl}$ and creatinine levels $<1.3 \mathrm{mg} / \mathrm{dl}$ have a good prognosis. Out of the 95 patients as sampled, there were $90(94.7 \%)$ cured, and $5(5.2 \%)$ patients died.
\end{abstract}

Keywords: Acute Post Streptococcal Glomerulonephritis, Prognostic Value, Ureum, Creatinine, Child

\section{Introduction}

Glomerulonephritis is a general term used to describe several kinds of kidney disease experienced of proliferation and inflammation at the glomerular because of the immuneologis process. ${ }^{1}$ Acute glomerulonephritis (AGN) is a group of symptoms marked by sudden decreased of glomerular filtration rate with clinic manifestation, such as edema, hematuria, hypertension, oligouria and renal insufficiency, and also called as acute nephritic syndrome (ANS). ${ }^{2}$ The most common AGN in children in developing countries is acute post streptococcal glomerulonephritis (APSGN) mostly in age of 3-8 years and male to female ratio of 2.3: 1 . $^{3}$ APSGN is mostly found in developing countries leading to acute kidney injury, and potentially increasing morbidity in children and threatening life if delayed diagnosis and subsequently inaccurate treatment.
Complications can occur in acute phase of the illness including acute kidney injury, acute pulmonary edema, and or hypertensive encephalopathy. ${ }^{4}$

Renal function can be measured by glomerular filtration rate (GFR) and in clinical practice, GFR is assessed by means of estimating creatinine or ureum clearance based on levels of serum ureum and creatinine. ${ }^{5,6}$ Consequently, ureum and serum creatinine may be used to detect outcome of children with APSGN. Therefore, it is important and necessary to carry out a study concerning the relationship between serum ureum and creatinine level and the course of illness and outcome of children with APSGN. ${ }^{7}$ Etuk et al. found the mean of serum creatinine levels of patients with APSGN was $1.5 \mathrm{mg} / \mathrm{dl}$ but the serum creatinine level is not a prognostic factor of APSGN. ${ }^{8}$ Other study in Iran by Sepahi et al., and Kumar concluded that decreased renal function influences the quality of life associated with morbidity and mortality of patients. ${ }^{4,9}$ Serum ureum and creatinine should be measeured 
earlier to investigate if the renal function of patients has deacreased and subseuqently treatment may be given earlier. Identification of prognostic factors may support a comprehensive treatment to patients in order to reduce morbidity and mortality.

This study was done to investigate the ureum and serum creatinine levels in children with APSGN in accordance with the outcome of patients.

\section{Materials and Methods}

This study is a retrospective cohort study on ureum and serum creatinine prognostic value in APSGN children. This study conducted in Dr. Wahidin Sudirohusodo hospital in Makassar, South Sulawesi by using a medical history of APSGN patient record in Department of Medical and Child Health from 2009-2013. We conducted the study in August until December 2014 using the medical record of APSGN children, which are treated from January until December 2013. During the study period, we have been identification the prognostic factor among 95 samples of APSGN patients' medical record in children. Before we did all the examination on patient, written informed consent was obtained from the patients' parents or their legal guardian following full and detail explanation regarding the study's protocol and this study was approved by the Ethics and Industry Research Committee of the hospital.

Those study samples are the medical history data of patients met the inclusion and exclusion criteria included into the study, it then observed till the samples are divided into two groups; the recovered and death group. The inclusion criteria are the entire APSGN patient's medical record treated in Dr. Wahidin Sudirohusodo hospital from 2009-2013. While, the exclusion criteria are the patients' suffering from congenital malformations associated with renal function and the patients who forced home. Every sample are noted such as patient initial, registered number, age on diagnosed, sex, nutritional status, blood ureum level, and blood creatinine level. All the data collected from the medical history are group based on the purposed and type of data, then analyzed using the appropriate statistical method, which are the univariate, bivariat (Unpaired Student's t test, Mann Whitney test, $X^{2}$ test (Chi-square) or Fisher's Exact test), and multivariate (logistic multiple regression analyses) analyses with value of not significant, when $p>0.05$, significant if $p \leq 0.05$, and very significant if $p<0.01$.

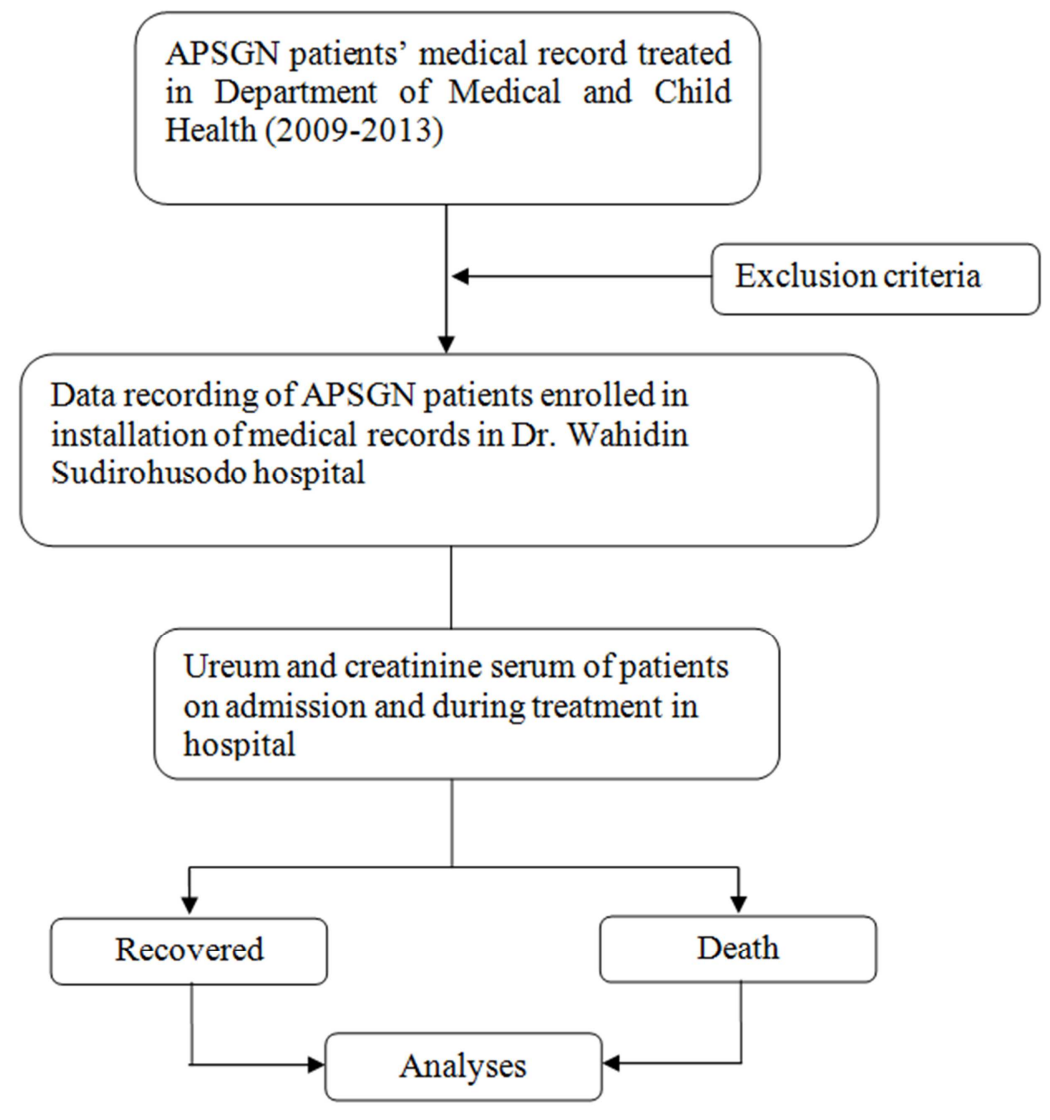

Figure 1. Study flow scheme.

\section{Results}

\subsection{Sample Characteristics}

Table 1 shows the sample characteristics assessed such as sex, nutritional status and patient's age. The total of study sample is 95 samples, consisting of $56(58.9 \%)$ male and 39 (41.1\%) female. Of all the samples, there are 40 patients of well nutritional $(42.1 \%)$ and 55 patients of under nutritional $(57.9 \%)$ based on the WHO parameters (patients' weight and 
height). ${ }^{12}$ Of the 95 study samples, it is divided into the recovered group is 9.56 years (median) and the death group group of recovered and death group. The patient's age of the

is 8.32 years (median).

Table 1. Distribution and analyses of characteristics on patient outcomes.

\begin{tabular}{|c|c|c|c|c|}
\hline Characteristics & Recovered n (\%) & Death n (\%) & Total n (\%) & Value $P$ \\
\hline Sex & & & & 0.961 \\
\hline Male & $53(58.8)$ & $3(60)$ & $56(58.9)$ & \\
\hline Female & $37(41.2)$ & $2(40)$ & $39(41.1)$ & \\
\hline Body Mass Index (BMI) & & & & 1.000 \\
\hline Well & $38(42.2)$ & $2(60)$ & $40(42.1)$ & \\
\hline Under & $52(57.8)$ & $3(40)$ & $55(57.9)$ & \\
\hline Mean & 9.54 & 7.24 & & \\
\hline$\pm \mathrm{SD}$ & 3.02 & 2.07 & & \\
\hline Range & $3.40-14.67$ & $3.80-8.80$ & & \\
\hline Early Ureum level (mg/dl) & & & & 0.003 \\
\hline Mean & 51.77 & 191.8 & & \\
\hline$\pm \mathrm{SD}$ & 48.67 & 135.36 & & \\
\hline Range & $12-266$ & $48-455$ & & \\
\hline Mean & 0.95 & 5.34 & & \\
\hline$\pm \mathrm{SD}$ & 0.89 & 8.55 & & \\
\hline Range & $0.2-5.3$ & $0.6-20.6$ & & \\
\hline
\end{tabular}

\subsection{Relationships Analyses of Study Samples and Outcomes Characteristics}

As shown in Table 1, the relationships of sample characteristic to the outcomes. There is no significant difference of sex $(p=0.961)$, BMI $(p=1.000)$, and age $(p=$ $0.108)$ to the outcomes. There is a significant difference of early ureum level to the early outcomes with value $p=0.003$ and the significant difference of early creatinine level to the outcomes with value $p=0.020$.

Figure 2 is a graph showing the decline in ureum levels of early admission in the hospital till the end of the recovered patients treatment period, statistically significant better of ureum levels were compared during treatment with $p=0.000$ and if analyzed from the beginning, during treatment, and ending with $p=0.000$. In the group of death associated with an increase of ureum levels of admission, during treatment and the end of treatment, but the results of the analyses showed that the increase was not statistically significant ( $p=$ $0.893 p=0.549$ ).

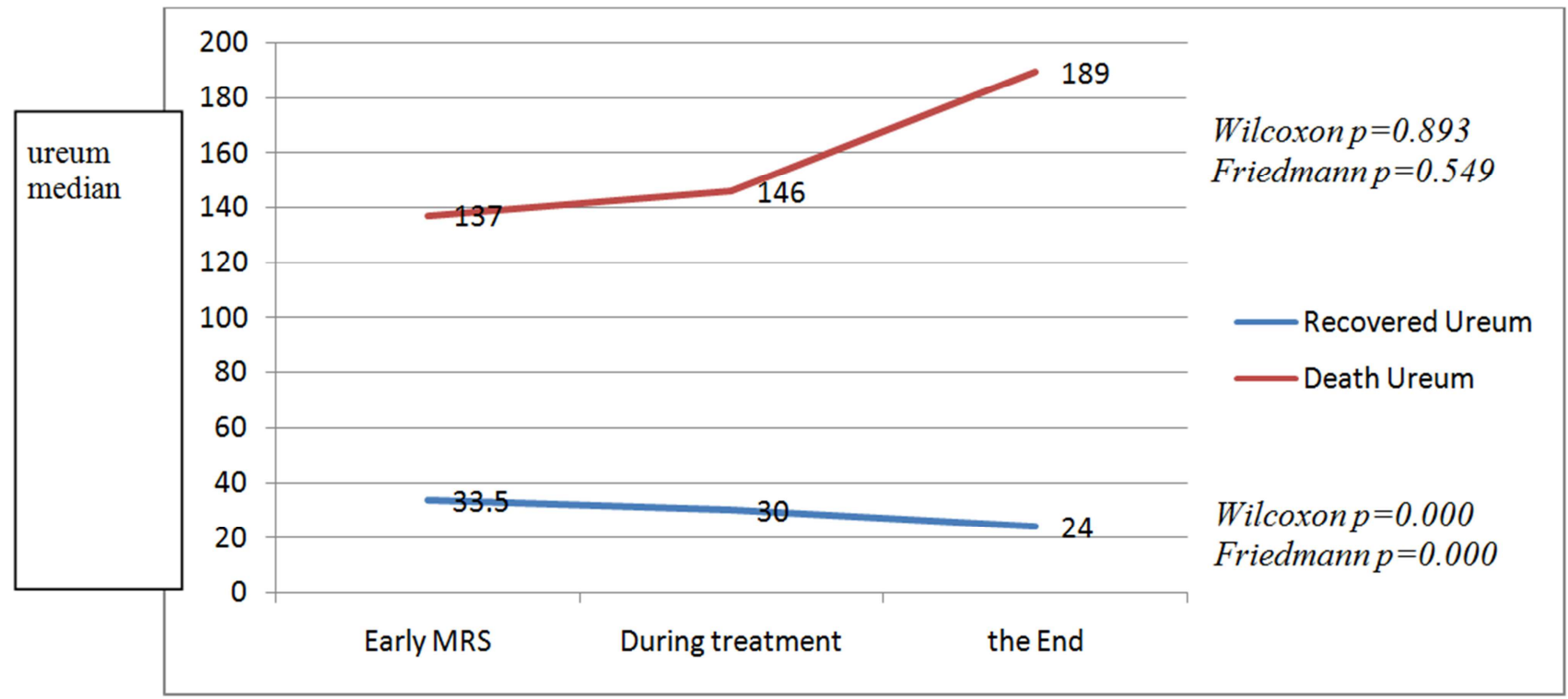

Figure 2. Graph changes in ureum levels at the early of hospitalized, during treatment and in the end of the recovered and death group.

Figure 3 is a graph showing the decrease in creatinine levels of early admission in hospital till the end of the treatment period in patients who recovered. The results of the analyses of the initial creatinine levels were compared with creatinine levels during treatment were statistically significant with $p=0.000$ similarly if analyzed from the 
beginning, during treatment, and in the end with a value of $p=0.000$. In the group of death obtained an increase in creatinine levels of early admission in hospital till the end of the treatment period in patients who death, but the results of the analyses of the initial creatinine levels were compared during treatment was not statistically significant with $p=$ 0.786 similarly if analyzed from beginning, during treatment, and in the end of treatment with a value of $p=0.074$.

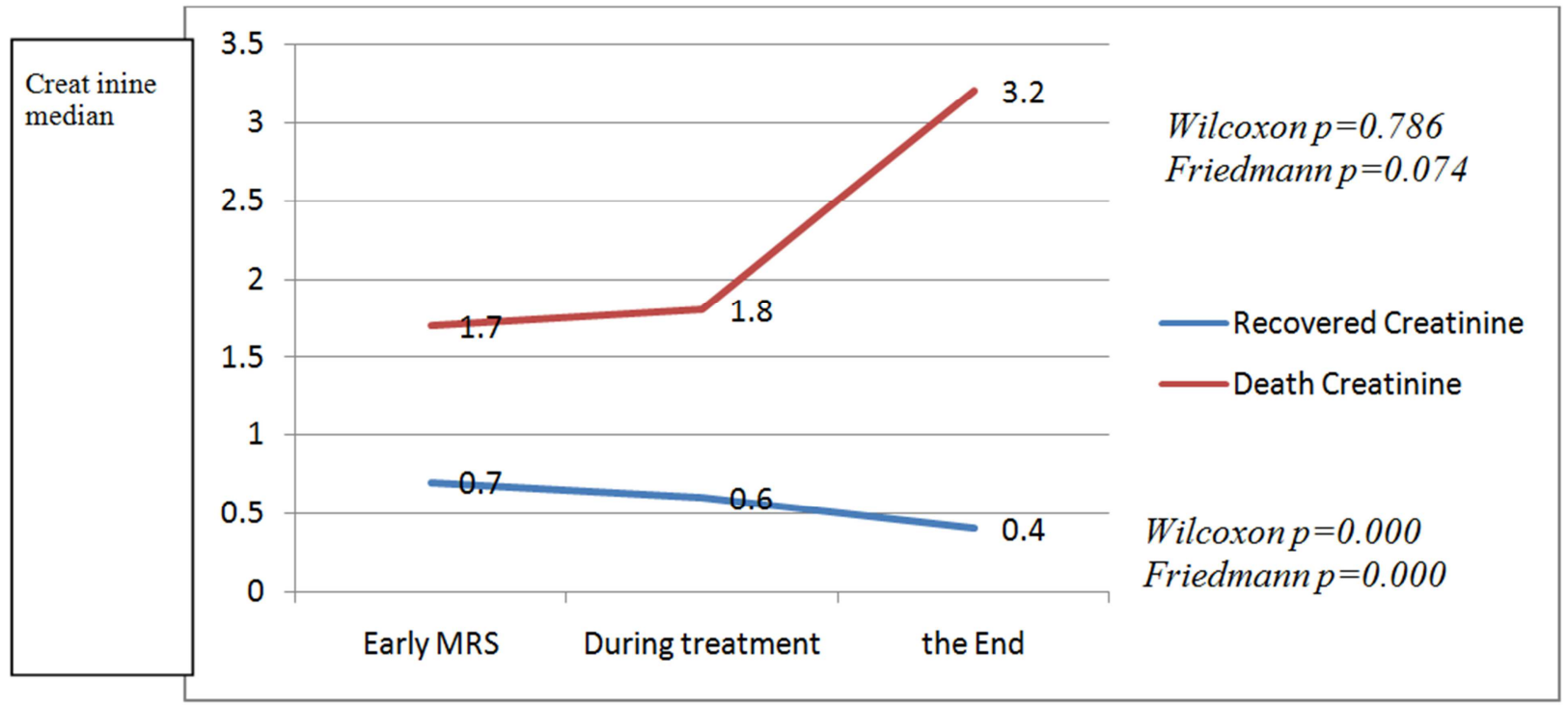

Figure 3. Graph changes in creatinine levels at the early of hospitalized, during treatment, and in the end of the recovered and death group.

\subsection{Identification of Independent Prognostic Factors to the Outcomes}

Table 2 shows the double logistic regression analyses of ureum and creatinine variable tested, the blood ureum level is a prognostic factor of independent to the outcomes of APSGN children $(p=0.032)$.

Table 2. The results of multiple logistic regression analyses of ureum and creatinine on patient outcomes in APSGN children.

\begin{tabular}{lllllll}
\hline No. & Variable & B & SE & Value $\boldsymbol{p}$ & OR & $\mathbf{9 5 \%}$ CI \\
\hline 1. & Ureum & 0.021 & 0.010 & 0.032 & 1.021 & $\begin{array}{l}1.002- \\
1.041\end{array}$ \\
2. & Creatinine & -0.144 & 0.260 & 0.579 & 0.860 & $\begin{array}{l}0.520- \\
1.441\end{array}$ \\
\hline
\end{tabular}

B: Regression coefficient SE: Standard error OR: odds ratio CI: confidence interval

In this study also found the ureum $119 \mathrm{mg} / \mathrm{dL}$ which have the greatest AUC (area under curve) value of 0.850 , sensitivity $90 \%$, specificity $80 \%$, positive predictive value $98 \%$ and negative predictive value of $30.76 \%$, with a value of $p=$ 0.001 , which means that the APSGN patients with ureum levels $<119 \mathrm{mg} / \mathrm{dL}$ had a tendency to recover by $98 \%$. The data of this study also showed a significant decrease in ureum levels in recovered patients $(p=0.000)$, and the death patient increase in ureum levels during treatment, although it is not significant, indicating the results of the statistical analyses ( $p=0.893$ and $p=0.549)$, it is explained that the ureum which has a tendency to decrease during treatment showed a good prognosis and conversely high levels of ureum with a tendency persists or increases during treatment prognosis is not good.

The statistical analyses obtained creatinine levels 1.3 $\mathrm{mg} / \mathrm{dL}$ with a great AUC as value 0.811 ; sensitivity $82.2 \%$, specificity $80 \%$, positive prediction value $98 \%$ and negative prediction value $20 \%$, with value $p=0.007$, which means the APSGN patient with creatinine levels $<1.3 \mathrm{mg} / \mathrm{dL}$ has tendency of recovered is $98 \%$.

\section{Discussion}

This study found that APSGN was more in male (58.9\%) than female (39\%) with ratio male: female is $1.4: 1$. Ho CZ and Chen $\mathrm{YH}$ in Taiwan also noted similar result with ratio 2.3:1. ${ }^{10}$ So do as the report in Nigeria and Iran with ratio $1.23: 1{ }^{8,9}$ In Indonesia, APSGN was more in male $(58.3 \%)$ than female $(41.7 \%){ }^{11}$ The reason may be due to male often being outside home and exposed to streptococcus beta hemoliticus group $A$ infection. ${ }^{7}$ The mean age of patient is 9.56 years with range of $3.4-14.67$ years but other showed the mean age of 6-8 years. Bingler et al. reported APSGN generally in children above 6 years whereas Ahn et al. obtanined lower in children under 2 years because of the immune complex system is still immature and pharyngitis incidence. ${ }^{5,7}$ Statistical analyses did not show a significant difference between age and APSGN patient outcomes ( $p=$ 0.108 ) and same as the report by Mossie and Shimels. ${ }^{3}$ However, Ahn and Suarta IK et al. concluded that older children as poor prognostic factor. ${ }^{6,7}$

Our study did not show a significant difference between nutritional status and outcomes $(p=1.000)$. Same result was reported by Mossie and Shimels in Ethiopia. ${ }^{3}$ On this study, we found a significant difference between the early serum ureum levels and patient's outcomes $(p=0.003)$ and the result was also found by Albar et al. reporting the increase of serum ureum and creatinine levels was $23.9 \%$ and $34.4 \%$, 
respectively. ${ }^{11}$

Based on the initial of ureum levels change during treatment, it is important to perform serial examinations ureum admission of patients at the beginning and during the treatment. However, it should be considered the influence of extra renal factors that may affect the ureum levels. ${ }^{5}$ Ureum levels of $119 \mathrm{mg} / \mathrm{dL}$ cut point included in this study is the initial ureum levels on admission were not able to describe the pure ureum as a result of renal excretion as it allows extra renal factors influence and cannot be removed before entering the hospital; therefore, prognostic determine not only to see the early ureum but patterns of change during treatment can help to determine the APSGN patient prognosis outcomes. Early creatinine statistic test result in recovered group showed a significant difference between the early creatinine levels and the outcomes $(p=0.020)$.

According to our study, the early creatinine levels significant decreased from entering the hospital until the end of treatment in recovered group. The early creatinine levels statistic analyses compared to creatinine levels during treatment has statistic difference with value $p=0.000$. Thus, if we analyze from the early, during treatment, and in the end of treatment with value $p=0.000$. While, in the death group obtained an increased of creatinine levels at the early until the end of treatment, but it is not significant $(p=0.786)$. It is also found that there is no significant difference in the early creatinine levels with a decreased tendency during treatment until the end of treatment $(p=0.074)$. Based on the data, the creatinine levels with decreased tendency during treatment has a good prognostic, otherwise the creatinine levels with persist or increased tendency during treatment has a poor prognostic. Thus, we have to do such creatinine levels serial examinations during the treatment to assess changes in creatinine levels and prognosis, although there is no statistically significant differences probably due to the death patient sample size is too small.

The results of logistic regression analyses showed that only the blood ureum levels can be an independent prognostic factor for outcome in APSGN children $(p=0.032)$. Similar results were reported by Mossie and Shimelis but Sepahi et al. and Kumar found increased creatinine levels influence the mortality of patient. ${ }^{3,4,9}$ The difference may be due to different study design and sample size. Limitations in our study was the use of secondary data from medical records, while strengthen of the study is cohort design and it may reflect the situation in Indonesia because of conducted at the national referral hospitals.

We conclude that cure rate of APSGN in children hospitalized in Dr. Wahidin Sudirohusodo Makassar was $94.7 \%$, ureum levels $<119 \mathrm{mg} / \mathrm{dL}$ and creatinine levels $<1.3$ $\mathrm{mg} / \mathrm{dL}$ indicating a good prognosis in APSGN patients. Ureum is an independent prognostic factor for outcome in APSGN patients. Ureum and creatinine levels which have a tendency to decrease during treatment have a good prognosis and levels of urea and creatinine which have a tendency to persist or increased during the treatment prognosis is poor. It is expected that this study can be used as one of the additional reference in handling the APSGN patient and we suggested a more comprehensive and serial examination of ureum levels and serum creatinine levels during treatment in hospital to anticipate the complications and mortality as well as the necessary collaborative prospective cohort study with a larger sample.

\section{References}

[1] Iturbe BR, Musser JM. The current state of poststreptococcal glomerulonephritis. J Am Soc Nephrol. 2008; 19: 1855-64.

[2] Wong W. Glomerulonephritis-acute in children. Starship Children's Health Clinical Guideline: 2009; 1-3.

[3] Mossie L, Shimelis D. Outcome of children with acute post streptococcal glomerulonephritis in Tikur Anbessa specialized teaching hospital. Ethiopian Journal of Pediatrics and Child Health. 2012; 8: 1-16.

[4] Kumar GV. Clinical study of post streptococcal glomerulonephritis in children with special reference to presentation. Current Pediatric Respirology; 2011; 15(2): 8992.

[5] Bingler MA, Ellis D, Moritz ML. "Acute post-streptococcal glomerulonephritis in a 14-month-old boy: why is this uncommon?". Pediatr Nephrol. 2007; 22 (3): 448-50

[6] Suarta IK. Erythrocyturia and proteinuria conversion in poststreptococcal acute glomerulonephritis. Paediatrica Indonesiana. 2006; 46(3-4): 71-6.

[7] Ahn SY, Ingulli E. Acute poststreptococcal glomerulonephritis: an update. Curr Opin Pediatr. Apr 2008; 20(2):157-62.

[8] Etuk IS, Anah MU, Eyong ME. Epidemiology and clinical features of glomerulonephritis in Calabar. Niger J Physiol Sci. 2009; 24(2): 91-4.

[9] Sepahi MA, Shajari A, Shakiba M. Acute glomerulonephritis: A 7 years follow up of children in Center of Iran. Acta Medica Iranica. 2011; 49(6): 375-8.

[10] Ho CZ, Chen YH. The clinical analyses of acute glomerulonephritis and neprotic syndrome in Chinese children. J Med Sci. 1995; 15 (6): 429-40.

[11] Albar H, Rauf S. The profile of acute glomerulonephritis among Indonesian children. Paediatrica Indonesiana; 2005; 45(11-12): 264-9. 